\title{
O que os pediatras confecem sobre afecções oculares na criança?
}

What do pediatricians know about children's eye diseases?

$\begin{array}{llll}\text { Michel } & \text { Broilo Manica } & 1 \\ \text { Zélia } & \text { Maria Silva } & \text { Corrêa } & { }^{2} \\ \text { Ítalo } & \text { Mundialino } & \text { Marcon } & 3 \\ \text { Nelson } & \text { Telichevesky } & 4 \\ \text { Luiz Fernando Loch } & 5\end{array}$

Estudo realizado no Serviço de Oftalmologia da Irmandade Santa Casa de Misericórdia de Porto Alegre ISCMPA e no Serviço de Pediatria do Hospital Materno Infantil Presidente Vargas - HMIPV.

${ }^{1}$ Residente do primeiro ano do Curso de Especialização do Serviço de Oftalmologia da Irmandade da Santa Casa de Misericórdia de Porto Alegre - ISCMPA.

${ }^{2}$ Médica Responsável pelos Setores de Retina e Oncologia Ocular da Irmandade da Santa Casa de Misericórdia de Porto Alegre - ISCMPA. Doutora em Oftalmologia pela Universidade Federal de São Paulo - UNIFESP.

${ }^{3}$ Médico Chefe do Serviço de Oftalmologia da Irmandade da Santa Casa de Porto Alegre - ISCMPA. Mestre e Doutor em Oftalmologia pela Universidade Federal de São Paulo - UNIFESP. Professor Livre-Docente da Disciplina de Oftalmologia da Fundação Faculdade Federal de Ciências Médicas de Porto Alegre - FFFCMPA.

${ }^{4}$ Médico Oftalmologista do Serviço de Estrabismo e Oftalmologia Infantil da Irmandade da Santa Casa de Misericórdia de Porto Alegre - ISCMPA. Hospital da Criança Santo Antônio.

${ }^{5}$ Médico Pediatra da Emergência Pediátrica do Hospital das Clínicas de Porto Alegre - HCPA. Coordenador da UTI Pediátrica do Hospital Universitário da Universidade Luterana do Brasil - ULBRA.

Endereço para correspondência: Av. Nilo Peçanha, $\mathrm{n}^{\circ} 2421$ Porto Alegre (RS) CEP 91330-001

E-mail-zmcorrea@terra.com.br

Recebido para publicação em 03.06.2002

Aceito para publicação em 07.10.2002

\section{RES UMO}

Objetivo: Avaliar o grau de conhecimento dos pediatras sobre os problemas oculares na criança, seu diagnóstico e conduta, por meio de questionário padronizado. Métodos: Aplicou-se questionário semi-estruturado, no qual constam dados demográficos e questões sobre conhecimentos oftalmológicos, entre pediatras na cidade de Porto Alegre no período de janeiro a abril de 2002. Após realizou-se estudo de delineamento transversal, cujas respostas foram tabuladas. Amostragem foi por conveniência. Foram consideradas erradas as questões em branco e as respostas dos testes foram comparadas a artigos já publicados. Resultados: Foram entrevistados 140 pediatras com idade média de 37,78 anos, e desses 89 $(63,57 \%)$ eram do sexo feminino. O tempo médio de profissão referido pelos entrevistados foi 11,77 anos. Em relação à análise das questões $(n=19)$, a média de acertos foi de 11(58\%). O grupo que teve melhor número de acertos foi o com menor tempo de atuação profissional em pediatria. A primeira escolha de antibiótico foi a tobramicina $(61 \%)(n=85)$, a segunda, o cloranfenicol $(31 \%)(n=44)$ e outros foram citados esporadicamente $(8 \%)$ $(n=11)$. Mais da metade dos entrevistados desconheciam o momento correto da avaliação oftalmológica na retinopatia da prematuridade. Grande parte dos pediatras entrevistados $(70 \%)(n=98)$ desconheciam o significado correto do termo ambliopia. Questões sobre catarata congênita, glaucoma congênito e leucocoria também revelaram o desconhecimento oftalmológico neste grupo de pediatras. Conclusão: Os resultados sugerem o pouco conhecimento oftalmológico entre os pediatras entrevistados.

Descritores: Conhecimentos, atitudes e prática; Pediatria; Questionários; Competência clínica; Qualidade dos cuidados de saúde; Oftalmopatias/diagnóstico

INTRODUÇÃO

Em 1992, a Organização Mundial de Saúde (OMS) estimava em 1,5 milhões o número de crianças cegas no mundo. Embora a cegueira infantil tenha uma baixa prevalência, sua magnitude é comparável à cegueira do adulto. Contudo, estudos realizados pela OMS referem que cerca de $75 \%$ dos casos de cegueira são passíveis de prevenção, o que implicaria uma melhora na qualidade de vida, diminuição considerável dos custos econômicos e sociais dos tratamentos especializados e programas de reabilitação.

Sabe-se que entre as causas gerais de cegueira previsível estão problemas oculares que ocorrem em crianças, tornando-se imprescindível a detecção imediata da baixa acuidade visual, o diagnóstico e tratamento precisos, e a estimulação visual precoce ${ }^{(1)}$.

A distribuição inadequada de oftalmologistas nas diferentes regiões do país transfere aos pediatras grande parcela do atendimento inicial dos pa- 
cientes. A pediatria, devido à sua importância no atendimento primário da criança, desenvolve um importante papel na prevenção à cegueira. Convém salientar que grande parte das doenças sistêmicas cursam com algum tipo de comprometimento ocular sendo necessário que todo médico tenha conhecimentos básicos de saúde ocular, que o capacitem à condução desses ca$\operatorname{sos}^{(2)}$

Cabe aos pediatras o diagnóstico e tratamento das afecções oculares mais simples, bem como o encaminhamento para o exame oftalmológico especializado quando for necessário(3). Devido a esta responsabilidade, existe uma preocupação por parte dos oftalmologistas quanto ao nível de conhecimento e esclarecimento dos pediatras nesta área.

O presente trabalho tem como objetivo mostrar o nível de conhecimento básico entre os pediatras, acerca das condições oftalmológicas mais prevalentes e importantes na infância, tanto para intervenção imediata quanto para orientação e encaminhamento do paciente.

\section{MÉTODOS}

Durante o mês de janeiro de 2002 realizamos um estudo piloto, com 30 pediatras na cidade de Porto Alegre. Uma análise posterior do instrumento utilizado apontou a necessidade de mudanças e o acréscimo de outros temas para uma melhor análise.

Esse novo questionário foi então aplicado no período de janeiro a abril de 2002, a 159 médicos com Título de Especialista em Pediatria (TEP), reconhecido pela Associação Médica Brasileira e fornecido pela Sociedade de Pediatria RS, em grandes hospitais como: Hospital de Clínicas de Porto Alegre (HCPA); Hospital Materno Infantil Presidente Vargas (HMIPV); Hospital São Lucas (PUC/RS); Hospital da Criança Santo Antônio - Santa Casa de Misericórdia de Porto Alegre e Hospital Luterano (ULBRA) e, ainda, em consultórios particulares na cidade de Porto Alegre.

Estima-se que 912 pediatras com Título de Especialistas em Pediatria atuem em Porto Alegre. O referido questionário foi aplicado por um dos autores (MBM), previamente treinado quanto à técnica de condução das entrevistas, tentando não influenciar nas respostas.

A amostragem foi por conveniência (não probabilística) e o número de pediatras entrevistados, estabelecido arbitrariamente $150( \pm 16 \%)$.

$\mathrm{O}$ estudo foi de delineamento transversal, cujo instrumento utilizado constou de uma parte inicial de dados demográficos a partir de informações como idade, sexo, tempo de atuação em pediatria, subespecialidade e tempo de atuação na subespecialidade. A segunda parte era constituída de duas perguntas com respostas abertas e dezessete testes de escolha simples, abrangendo problemas oftalmológicos no recémnascido, o uso de colírios com antibióticos, encaminhamento precoce de crianças para avaliação oftalmológica, estrabismo, ambliopia, avaliação da visão em criança pré-verbal, retinopatia da prematuridade, efeito colateral dos corticoesteróides, catarata congênita, leucocorias, obstrução lacrimal congênita e glaucoma congênito.
Os entrevistados foram orientados a não mudarem as respostas já assinaladas. As entrevistas individuais tiveram duração de 10 a 30 minutos.

O projeto de pesquisa recebeu aprovação do Comitê de Ética em Pesquisa do HMIPV/CEP, reunido em 09/01/2002. Obteve-se consentimento de todos participantes que preencheram o questionário para usar os dados nesse trabalho.

Os questionários respondidos foram analisados em conjunto, já que esta pesquisa não visa avaliar a atuação dos profissionais dos locais específicos, mas o conhecimento geral desses. Questões deixadas em branco foram consideradas erradas. São apresentados dados percentuais sobre respostas certas ou erradas.

\section{RESULTADOS}

Dos 159 pediatras entrevistados, 19 (11,35\%) não quiseram responder o questionário por não se considerarem preparados ou "não terem tempo para tal tarefa". Considerando-se os 140 pediatras que responderam ao questionário, $89(63,57 \%)$ foram do sexo feminino, com idade média 37,78 anos desvio padrão $(\mathrm{DP})=8,6$ anos. O tempo médio de profissão dos pediatras foi de 11,77 anos $\mathrm{DP}=8,27$ anos.

Em relação à análise das 19 (dezenove) questões, 17 (dezessete) foram de escolha simples e 2 (duas) questões abertas. A média de acertos das questões foi 11 (58\%) com DP =2,06.

$\mathrm{O}$ grupo com tendência a apresentar maior número de acertos foi composto pelos profissionais com menor tempo de atuação, conforme percebemos na tabela 1 .

Em relação às respostas observamos:

- $60 \%(n=84)$ dos entrevistados encaminham corretamente as crianças para avaliação oftalmológica antes dos dois anos.

- $45 \%(\mathrm{n}=63)$ desconhecem a conduta correta quando o recém-nascido apresenta secreção ocular.

- A primeira escolha de antibiótico tópico (colírio) entre os pediatras foi a tobramicina $(61 \%)(n=85)$, a segunda foi o cloranfenicol $(31 \%)(n=44)$ e outros foram citados esporadicamente $(8 \%)(n=11)$. A medicação foi prescrita por $47 \%(n=66)$ dos entrevistados de $2 / 2 \mathrm{~h}$. Em $45 \%$ (n=63) dos casos tempo médio de 7 dias.

- $15 \%(\mathrm{n}=21)$ dos entrevistados não sabem o manejo adequado de criança com estrabismo.

\begin{tabular}{|c|}
\hline $\begin{array}{l}\text { Tabela 1. Tempo de atuação na área pediátrica comparado com } \\
\text { a média de cada grupo }\end{array}$ \\
\hline 1 - 5 anos \\
\hline $5,1-10$ anos \\
\hline mais de 10 anos \\
\hline $\begin{array}{l}\text { Nota: Tempo de pediatria }=\text { tempo de exercício da profissão médica na área de } \\
\text { pediatria (em anos); Média do } n^{0} \text { de acertos = média de respostas certas no } \\
\text { determinado grupo de pediatras; DP = desvio padrão das respostas certas no } \\
\text { grupo de pediatras }\end{array}$ \\
\hline
\end{tabular}


- $70 \%$ dos entrevistados $(n=98)$ desconheciam que o termo ambliopia refere-se a redução da acuidade visual importante.

- $13 \%$ dos pediatras $(n=18)$ não sabiam como avaliar a visão da criança pré-verbal.

- $11 \%$ dos testados $(n=15)$ não perguntavam sobre a história familiar ocular na anamnese.

- 20\% (n=28) dos entrevistados desconheciam a melhor idade para iniciar o tratamento da baixa acuidade visual.

- 53\% $(n=74)$ desconheciam o momento correto da avaliação oftalmológica na retinopatia da prematuridade.

- 30\% ( $n=42)$ desconheciam que os corticoesteróides, usados abusivamente, podiam causar aumento da pressão ocular e catarata entre outras doenças.

- $36 \%(n=50)$ não sabiam a conduta inicial na catarata congênita e $75 \%(n=105)$ não sabiam informar às famílias de seus pacientes sobre o prognóstico do tratamento dessa doença.

- $63 \%(n=88)$ não lembravam que retinoblastoma, retinopatia da prematuridade e doenças exsudativas da retina são causas de leucocoria e $10 \%(n=14)$ não sabiam que o retinoblastoma é maligno.

- $65 \%$ (n=91) não sabiam o momento correto para encaminhar ao oftalmologista criança com obstrução lacrimal congênita.

- 52\% $(\mathrm{n}=73)$ não sabiam que a tríade sintomática clássica do glaucoma congênito é fotofobia, lacrimejamento e blefaroespasmo.

\section{DISCUSSÃO}

Os resultados apresentados sugerem deficiência do conhecimento oftalmológico entre os pediatras, já que o índice de acertos nas 19 (dezenove) questões propostas foi inferior ao esperado. Baseando-se na porcentagem de profissionais que não quiseram responder ao questionário $(11,35 \%$ da amostra total entrevistada), supomos que os resultados poderiam ser subestimados. A desinformação dos pediatras na área de oftalmologia provavelmente se deve à pouca ênfase nesta área durante a formação acadêmica e residência médica ${ }^{(3)}$. É importante salientar que o questionário é um instrumento teórico que pode avaliar de maneira tendenciosa os conhecimentos dos pediatras.

Guinguerra et al demonstraram conhecimento insuficiente entre alunos de Medicina do $6^{\circ}$ ano, encontrando falhas importantes no conhecimento básico de condutas simples, sugerindo uma inadequação dos cursos de graduação em prover o conhecimento mínimo esperado ${ }^{(4)}$. Porém, a maior porcentagem de acertos foi entre os pediatras com menor tempo de profissão, o que sugere uma possível melhora do curso médico. Devemos ressaltar que a oftalmologia teve um grande avanço nos últimos anos, justificando talvez a dificuldade dos pediatras atuantes há mais tempo, em acompanhar essa evolução. Supomos que o fato de não haver cursos de reciclagem oftalmológica para pediatras também justifique esse problema.

Quando comparamos nossos resultados demográficos - 89 $(63,57 \%)$ pertenciam ao sexo feminino, a idade média 37,78 anos DP $=8,6$ anos, com tempo médio de pediatria de 11,77
$\mathrm{DP}=8,27$ anos. $\mathrm{O}$ que parece mostrar uma procura maior do sexo feminino por esta área da Medicina.

A prescrição de colírios feita pelos pediatras com pouco conhecimento oftalmológico torna-se arriscada, pois os corticóides tópicos podem induzir com o longo tempo de uso a doenças como glaucoma e catarata entre outras ${ }^{(5)}$. O cloranfenicol não deve ser usado para tratamento de infecções menores - tanto por via sistêmica como tópica - pois este pode causar depressão da medula óssea e anemia aplástica ${ }^{(6)}$. A ocorrência dessas complicações é extremamente pequena. Também está contra-indicado o uso de cloranfenicol em pacientes com história familiar ou pessoal de falência hematopoiética relacionada a essa droga ${ }^{(7)}$.

Trabalhos publicados no Brasil, mostram inúmeras afecções oculares em crianças que demoram a serem diagnosticadas e encaminhadas para o oftalmologista. Deve-se lembrar a importância do estímulo visual adequado na infância (principalmente até os 6 anos), cuja privação leva à baixa da acuidade visual permanente ${ }^{(8)}$.

Ambliopia é o termo utilizado para definir baixa da acuidade visual bi ou unilateral (a mais freqüente), sem lesão orgânica aparente. Suas principais causas são estrabismo, anisometropia, altas ametropias não corrigidas precocemente e deprivação visual por falta de estímulo ${ }^{(9)}$. É uma situação clínica comum em crianças e seu diagnóstico e tratamento precoces trazem resultados satisfatórios ${ }^{(10)}$

Nossos resultados mostram que 36\% $(n=50)$ desconheciam a conduta na catarata congênita e $75 \%(n=105)$ não sabiam informar sobre o prognóstico do tratamento dessa doença. A indicação precoce da cirurgia da catarata congênita, o uso da correção óptica e tratamento com oclusão podem prevenir a ambliopia ${ }^{(10)}$, por isso a importância do encaminhamento precoce para o oftalmologista. O prognóstico da catarata congênita, diferente da cirurgia no adulto que proporciona excelentes resultados visuais em todo mundo, raramente apresenta bons resultados cirúrgicos, mostrando que o esclarecimento dessa doença aos pais possa proporcionar uma melhor adesão ao tratamento.

Dos entrevistados $63 \%(n=88)$ não sabiam que retinoblastoma, retinopatia da prematuridade e doenças exsudativas da retina são causas de leucocoria e $10 \%(n=14)$ não sabiam que o retinoblastoma é maligno. Leucocoria (ou pupila branca) é um sinal que pode causar confusão no diagnóstico diferencial, devido ao grande número de doenças oculares que envolvem esse sinal ${ }^{(11)}$. O esclarecimento rápido desse sinal é importante, pois o retinoblastoma, tumor intra-ocular maligno mais comum da infância, não tem predileção racial ou por sexo, apresenta-se bilateralmente em $25-35 \%$ dos casos e é em média diagnosticado por volta dos 18 meses de idade ${ }^{(12)}$. Há história familiar em cerca de $6 \%$ dos pacientes, predominando a herança autossômica dominante com penetrância incompleta, o que ressalta a importância do aconselhamento genético ${ }^{(12)}$. Em nosso país, tem risco de mortalidade ainda alto e seu tratamento precoce (radical ou conservador) é de fundamental importância no prognóstico e sobrevida dos pacientes. 
Aproximadamente metade dos pediatras entrevistados $(52 \%)(n=73)$ desconhecia que a tríade sintomática clássica do glaucoma congênito é fotofobia, lacrimejamento e blefaroespasmo. O glaucoma congênito primário também é uma causa de cegueira importante na infância. O diagnóstico precoce é o principal fator relacionado ao sucesso terapêutico e prognóstico visual dessa doença ${ }^{(13)}$.

Os achados deste estudo podem facilmente ser projetados em nível nacional. Portanto, parece ser de fundamental importância a incorporação de aulas básicas de oftalmologia nas Residências e Cursos de especialização de pediatria bem como uma maior interação multidisciplinar. Discussões de casos e cursos de atualização podem ser outro caminho para difundir conhecimentos de oftalmologia entre os pediatras. Não podemos esquecer a importância do ensino oftalmológico no currículo de Medicina, a fim de que tanto os pediatras como os médicos não especialistas estejam preparados para diagnosticar, orientar e encaminhar afecções oculares importantes na infância ou em qualquer idade.

\section{CONCLUSÃO}

Os conhecimentos oftalmológicos informados pelos pediatras entrevistados mostraram-se insuficientes para diagnosticar e tratar patologias oculares prevalentes na infância, bem como orientar os pais ou familiares dessas crianças.

\section{A B S T RAC T}

Purpose: To determine how much pediatricians know about eye disorders in children, their diagnosis and therapy, through a standardized questionnaire. Methods: A semistructured questionnaire with demographic data and questions about knowledge in ophthalmology among pediatricians was applied in the city of Porto Alegre between January and April 2002. Subsequently, a transverse delineation study was carried out and the answers were recorded. Blank answers were considered wrong. The answers were analyzed and compared to previously published articles. Results: The study group was composed of 140 pediatricians, of which $89(63.57 \%)$ were women. The average age of the group was 37.78 years. The average time of reported practice was 11.77 years. The average number of correctly answered questions was 11 (58\%). The group that scored best was composed of the physicians with less time of practice in pediatrics. The most used antibiotic was tobramicine $(61 \%)(n=85)$ followed by chloramphenicol $(31 \%)(n=44)$ and other classes of antibiotic were rare $(8 \%)$ $(n=11)$. The majority of the interviewed doctors did not know the correct time for ophthalmic evaluation in children with retinopathy of prematurity. Also, the majority of the interviewed $(70 \%)(n=98)$ ignored the correct meaning of ambliopia. Questions on congenital cataract, congenital glaucoma and leucocoria also revealed the lack of ophthalmic knowledge in the group of pediatricians. Conclusion: These data suggest deficient knowledge of ophthalmology among the interviewed pediatricians.

Keywords: Knowledge, attitudes, practice; Pediatrics; Questionnaires; Clinical competence; Quality of health care; Eye diseases/diagnosis

\section{REFERÊNCIAS}

1. Tartarella BM, Nakano K, Castro MTC, Martins MPA. Visão subnormal em crianças. Arq Bras Oftalmol 1991;54:221-4.

2. Cardoso de Melo O. Objetivos mínimos de um curso de oftalmologia em nível de graduação. Rev Bras Oftalmol 1978;37:213-8.

3. Manso PG, Ribeiro MD, Belfort Jr R, Chinen NH, Niero AL, Ribeiro CR. Avaliação de conhecimento oftalmológico entre pediatras. Arq Bras Oftalmol 1988;51:74-6.

4. Ginguerra AM, Ungaro ABS, Villela FF, Kara-José AC, Kara-José N. Aspectos do ensino de graduação em oftalmologia. Arq Bras Oftalmol 1998;61:546-50.

5. Moreira JBC. Iatrogenia ocular. Arq Bras Oftalmol 1987;50:172-4.

6. Flaunfelder FT, Bagby GC, Kelly DJ. Fatal aplastic anemia following topical application of ophthalmic chloramphenicol. Am J Ophthalmol 1982;93:356-60.

7. Nishi M, Vita Sobrinho JB. Efeitos colaterais sistêmicos da medicação tópica. In: Vita Sobrinho JB. Farmacologia \& terapêutica ocular. Rio de Janeiro: Cultura Médica; 1999. p. 360-6.

8. Beardsell R, Clarke S, Hill M. Outcome of occlusion treatment for amblyopia. J Pediatr Ophthalmol Strabismus 1999;36:19-24.

9. Cruz AAV. Ambliopia. Arq Bras Oftalmol 1991;54:139-47.

10. Lucena AR, Cantanhede TM, Trigueiro SA, Tavares S, Ventura LO. Freqüência e causas de ambliopia em pacientes assistidos na Fundação Altino Ventura - Recife - PE. Rev Bras Oftalmol 2001;60:50-4.

11. Gonçalves EA, Araújo HA, Carletti JR, Gonçalves JO. Leucocorias na clínica oftalmológica do Hospital Getúlio Vargas. Arq Bras Oftalmol 1988;51:77-9.

12. Santo RM, Bechara SJ. Tumores intra-oculares. Arq Bras Oftalmol 1998;61: 242-55.

13. Oliveira TL, Fulco GD, Monte JM. Glaucoma congênito: aspectos epidemiológicos e resultado cirúrgico. Rev Bras Oftalmol 1999;58:325-8

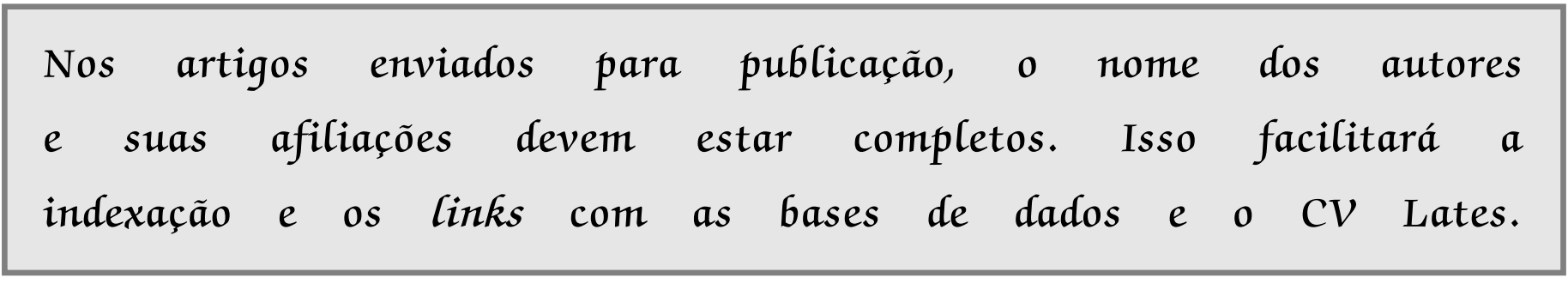

